\title{
Design of Ultra Wideband Phase Shifter with Improved Scattering Parameter Performances
}

\author{
Dyg Norkhairunnisa Abang Zaidel \\ Department of Electrical and Electronic Engineering, \\ Faculty of Engineering, Universiti Malaysia Sarawak, \\ 94300 Kota Samarahan, Sarawak, Malaysia. \\ azdnorkhairunnisa@unimas.my
}

\author{
Sharul Kamal Abdul Rahim, Norhudah Seman and \\ Raimi Dewan \\ Wireless Communication Centre \\ Faculty of Electrical Engineering, Universiti Teknologi \\ Malaysia, 81310 Johor Bahru, Johor, Malaysia \\ sharulkamal@fke.utm.my, huda@fke.utm.my, \\ raimidewan@gmail.com
}

\begin{abstract}
Aiming to achieve Ultra Wideband (UWB) performance, this paper presents an improvement of the squareshaped phase shifter design, which covers from $2.8 \mathrm{GHz}$ to 10.6 $\mathrm{GHz}$ by implementing step impedances in order to improve the impedance matching of the phase shifter, whilst leads to better scattering parameter results. The phase shifter design is formed by using multilayer technique where 3 layers of conductors are interleaved with 2 layers of substrates between each of the conductor's layer. This technique ensures the phase shifter design to be small in size. Computer Simulation Technology (CST) Microwave Studio is used in developing the design and optimization stage. Meanwhile the measurement is done by using Vector Network Analyzer (VNA). The square-shaped phase shifter with step impedances enhances the bandwidth up to $34 \%$ as compared to the conventional square-shaped phase shifter. Additionally, analysis on air gap and alignment is also presented in order to show how both conditions affect the performance of the proposed phase shifter design.
\end{abstract}

Keywords - enhanced bandwidth; multilayered technology; phase shifters; step impedances; ultra wideband.

\section{INTRODUCTION}

Phase shifter has been utilized in several microwave applications such as in sub-component of Butler Matrix (BM) [1], phase modulators and phased array antenna [2-4]. Phase shifter provides a constant phase difference between the outputs of two ports over designated frequency range. To date, few types of phase shifter have been reported by researchers. Since the allocation of UWB frequency range by Federal Communications Commission (FCC) in February 2002 [5], more research have been conducted on the design of microwave devices including phase shifters, couplers [6-8], filters [9], power amplifiers [1011] and also antenna [12-13] that capable to operate for UWB applications.

As reported in [14], a phase shifter has been designed by implementing two stubs; short circuited and open circuited at both ends of the microstrip lines. The combination of both stubs offer an approximately constant phase difference between output ports. However, the conducting pin that has been introduced in this design has led to the problem, where its capability to be realized in durable and hard substrate has been disputed. Therefore, to overcome the problem, the conducting pin has been replaced with slots to the ground plane [15].
A design of multilayered phase shifter has been introduced in [16]. The proposed phase shifter employed a short-ended stub onto the microstrip patch in order to expand the phase shift of the phase shifter to $270^{\circ}$. However, the stub has produced larger size phase shifter.

Another design of UWB phase shifter design reported by Abbosh in [17] has good agreement between the simulated and measured results over UWB frequency range. However, it is essential to study other alternative microstrip patch shapes that can be used as the patch stub rather than the ellipse-shaped phase shifter.

Therefore, in [18], the authors have introduced the squareshaped phase shifter. The size of the microstrip patch was reported to be smaller than ellipse-shaped phase shifter by $27 \%$. However, the performance is limited for the frequency range from 5.38 to $10.6 \mathrm{GHz}$ only. To overcome the limitation of the scattering parameter of the proposed phase shifter in [18], an improved design of square-shaped phase shifter is presented in this paper. The use of multilayer technique successfully in realizing a UWB phase shifter design with broad coupling and reduced in size. The addition of the step impedances [19] onto the square microstrip patch stub resulted in a $45^{\circ}$ phase shifter with phase deviation of $\pm 6.4^{\circ}$ from $2.8 \mathrm{GHz}$ to $10.6 \mathrm{GHz}$ and improved scattering parameter performances. The simulation shows that the return loss is better than $10 \mathrm{~dB}$ while insertion loss is $-1 \mathrm{~dB} \pm 0.35 \mathrm{~dB}$ within the designated band. The squareshaped phase shifter with step impedances enhances the coverage of the bandwidth up to $34 \%$ when compared to the square-shaped phase shifter without the steps as proposed in [18].

The proposed phase shifter is compared to the related designs reported in [14], [16-18] in Table 1. Based from the table, the proposed phase shifter gives broader bandwidth performance which is $7.8 \mathrm{GHz}$. The proposed phase shifter has smaller size compared to phase shifter proposed in [14], [16-17]. Even though the proposed phase shifter is larger in size compared to the phase shifter proposed in [18], the bandwidth coverage performance is better which makes the proposed phase shifter in this paper better compared to phase shifter proposed in [14], [16-18]. 